\title{
Chemoselective and Catalytic Trimethylsilylation of Alcohols and Phenols by 1,1,1,3,3,3-Hexamethyldisilazane and Catalytic Amounts of $\mathrm{PhMe}_{3} \mathrm{~N}^{+} \mathrm{Br}_{3}^{-}$
}

\author{
Arash GHORBANI-CHOGHAMARANI", Nasrin CHERAGHI-FATHABAD \\ Department of Chemistry, Faculty of Science, Ilam University, Ilam 69315516, Iran
}

\begin{abstract}
An efficient procedure for the trimethylsilylation of alcohols and phenols is presented. The combination of 1,1,1,3,3,3-hexamethyldisilazane and a catalytic amount of phenyltrimethylammonium tribromide $\left(\mathrm{PhMe}_{3} \mathrm{~N}^{+} \mathrm{Br}_{3}{ }^{-}\right)$was found to be effective for the trimethylsilylation of alcohols and phenols. The protection reaction is very simple and homogenously performed in dichloromethane at room temperature and mild conditions.
\end{abstract}

Key words: phenyltrimethylammonium tribromide; alcohol; phenol; protection; trimethylsilylation; hexamethyldisilazane

CLC number: $\mathrm{O} 643$

Document code: A

Synthetic methodology, as the building block of organic synthesis, continuously seeks new reagents, better reaction conditions, and more efficient and selective methods [1]. When a chemical reaction is to be carried out selectively at one reactive site in a multifunctional compound, the other reactive sites should be temporarily blocked [2]. Silyl ethers are the most popular protecting groups for alcohols and phenols in synthetic organic chemistry and various types of silyl ethers have been reported [3-5]. Trimethylsilylation is routinely used to protect alcohols and phenols, especially in steroids, sugars and natural product synthesis [6]. The silylation of alcohols is an important process not only as a method to protect alcohols but also for the synthesis of functional organosilicon compounds [7]. Generally, silyl ethers can be synthesized by the reaction of alcohols and phenols with hexamethyldisilazane [8-13], hydrosilanes [14], disilanes [15], alkylsilanes [16], allylsilanes [17], and trimethylsilyl azide [18] in the presence of a suitable catalyst. However, some of these procedures are not adequate for the chemoselective protection of the hydroxyl group for several reasons such as low selectivity, long reaction time, low product yield, toxicity, delicate purification, and lack of reactivity or difficulty in removing by-products. 1,1,1,3,3,3Hexamethyldisilazane (HMDS) as a cheap, stable, and commercially available reagent is one of the most widely used silylating agents for the trimethylsilylation of alcohols and phenols. Its handling does not require special precautions and the reaction workup is not time-consuming be- cause the by-product of the reaction is ammonia, which is simple to remove from the reaction media. However, the low silylating power of HMDS is the main drawback for its application. Therefore, to activate this reagent an appropriate catalyst is required.

\section{Experimental}

All chemicals were purchased from Fluka, Merck, or Aldrich chemical companies. The oxidation products were characterized by a comparison of their spectral (IR, ${ }^{1} \mathrm{H}$ NMR or ${ }^{13} \mathrm{C}$ NMR) and physical data with authentic samples. Phenyltrimethylammonium tribromide $\left(\mathrm{PhMe}_{3} \mathrm{~N}^{+} \mathrm{Br}_{3}{ }^{-}\right)$ is a commercially available material and was purchased from Merck.

The trimethylsilylation of 2-hydroxy-1,2-diphenylethanone (1j) with HMDS catalyzed by $\mathrm{PhMe}_{3} \mathrm{~N}^{+} \mathrm{Br}_{3}^{-}$as a typical procedure is outline below. To a mixture of $\mathbf{1 j}(0.212$ $\mathrm{g}, 1 \mathrm{mmol})$ and hexamethyldisilazane $(0.323 \mathrm{~g}, 2 \mathrm{mmol})$ in $\mathrm{CH}_{2} \mathrm{Cl}_{2}(10 \mathrm{ml}), \mathrm{PhMe}_{3} \mathrm{~N}^{+} \mathrm{Br}_{3}^{-}(0.018 \mathrm{~g}, 0.05 \mathrm{mmol})$ was added and the mixture was stirred at room temperature for $91 \mathrm{~min}$ (reaction progress monitored by thin layer chromatography). The reaction was then quenched with water (10 $\mathrm{ml}$ ), and the organic phase was dried over $\mathrm{Na}_{2} \mathrm{SO}_{4}(3 \mathrm{~g})$ and filtered after $10 \mathrm{~min}$. Evaporation of dichloromethane gave 1,2-diphenyl-2-(trimethylsilyloxy)ethanone $(0.276 \mathrm{~g}, 97 \%)$ as a white crystalline solid; m.p. $119-122{ }^{\circ} \mathrm{C} ;{ }^{1} \mathrm{H}$ NMR $(90$ $\left.\mathrm{MHz}, \mathrm{CDCl}_{3}\right) \delta 8.00(\mathrm{~m}, 2 \mathrm{H}), 7.36-7.51(\mathrm{~m}, 8 \mathrm{H}), 5.92(\mathrm{~s}$,

Received date: 26 March 2010.

*Corresponding author. Tel: +98-841-2227022; Fax:+98-841-2227022; E-mail: arashghch58@yahoo.com, a.ghorbani@mail.ilam.ac.ir

Foundation item: Supported by the research faculties of Ilam University, Ilam, Iran.

English edition available online at ScienceDirect (http://www.sciencedirect.com/science/journal/18722067). 
1H), 0.15 (s, 9H); IR (nujol, $\mathrm{cm}^{-1}$ ): v $1687,1597,1578$, 1 458, 1 377, 12511 109, 979, 888, 844, 698.

Trimethyl(phenethoxy)silane (2d). ${ }^{1} \mathrm{H}$ NMR (90 MHz, $\left.\mathrm{CDCl}_{3}\right) \delta 7.29(\mathrm{~s}, 5 \mathrm{H}), 3.85(\mathrm{t}, 2 \mathrm{H}), 2.90(\mathrm{t}, 2 \mathrm{H}), 0.14(\mathrm{~s}$, 9H); IR (KBr, cm $\left.{ }^{-1}\right): v 3$ 029, 2 956, 1 605, 1 497, 1454 , $1251,1095,929,883,841,748,698$.

Trimethyl(cholesteroloxy)silane (2k). ${ }^{1} \mathrm{H}$ NMR $(90 \mathrm{MHz}$, $\left.\mathrm{CDCl}_{3}\right) \delta 5.34(\mathrm{~m}, 1 \mathrm{H}), 3.49(\mathrm{~m}, 1 \mathrm{H}), 0.67-2.17(\mathrm{~m}, 43 \mathrm{H})$; ${ }^{13} \mathrm{C} \mathrm{NMR}\left(25 \mathrm{MHz}, \mathrm{CDCl}_{3}\right) \delta 141.4,121.3,72.4,56.9,56.4$, $50.4,42.8,42.4,39.9,39.6,37.5,36.6,36.3,35.9,32.0$, $28.3,28.0,24.4,24.0,22.8,22.6,21.2,19.4,18.8,11.9$, 0.36; IR (nujol, $\mathrm{cm}^{-1}$ ): v 2 853, $1464,1456,1378,1249$, $1085,896,840$.

Trimethyl(2-admantanoxy)silane (2l). ${ }^{1} \mathrm{H}$ NMR (90 MHz, $\left.\mathrm{CDCl}_{3}\right) \delta 3.78(\mathrm{~m}, 1 \mathrm{H}), 1.35-2.25(\mathrm{~m}, 14 \mathrm{H}), 0.08(\mathrm{~s}, 9 \mathrm{H})$; IR (nujol, $\mathrm{cm}^{-1}$ ): $v 2853,1$ 450, 1 354, 1 249, 1 133, 1093 , $880,839,752$.

Trimethyl(4-chlorophenoxy)silane (2o). ${ }^{1} \mathrm{H}$ NMR (200 $\left.\mathrm{MHz}, \mathrm{CDCl}_{3}\right) \delta$ 6.76-7.20 (dd, 71.5, 8.5, Hz, 4H), 0.02 (s, $9 \mathrm{H}) ;{ }^{13} \mathrm{C}$ NMR $\left(50 \mathrm{MHz}, \mathrm{CDCl}_{3}\right) \delta 156.7,129.5,122.8$, 117.3, 2.3.

Trimethyl(2-methoxy-4-methylphenoxy)silane (2r). ${ }^{1} \mathrm{H}$ NMR (200 MHz, $\left.\mathrm{CDCl}_{3}\right) \delta 6.70-6.84(\mathrm{~m}, 3 \mathrm{H}), 3.87$ (s, 3H), $2.36(\mathrm{~s}, 3 \mathrm{H}), 0.34(\mathrm{~s}, 9 \mathrm{H}) ;{ }^{13} \mathrm{C} \mathrm{NMR}\left(50 \mathrm{MHz}, \mathrm{CDCl}_{3}\right) \delta$ 150.6, 142.2, 131.4, 121.1, 120.6, 113.1, 55.5, 21.1, 0.6.

Trimethyl(4-benzylphenoxy)silane (2s). ${ }^{1} \mathrm{H}$ NMR (200
$\left.\mathrm{MHz}, \mathrm{CDCl}_{3}\right) \delta 6.95-7.59(\mathrm{~m}, 9 \mathrm{H}), 4.18(\mathrm{~s}, 2 \mathrm{H}), 0.57(\mathrm{~s}$, $9 \mathrm{H}) ;{ }^{13} \mathrm{C} \mathrm{NMR}\left(50 \mathrm{MHz}, \mathrm{CDCl}_{3}\right) \delta 153.7,141.7,134.3$, $130.2,129.1,128.8,126.3,120.2,41.3,0.53$.

\section{Results and discussion}

As a continuation of our previous studies on the application of new reagents and catalysts in organic functional group transformations [19-26], we now disclose a new, efficient, and mild procedure for the trimethylsilyl protection of a wide range of alcohols and phenols using HMDS in the presence of catalytic amounts of $\mathrm{PhMe}_{3} \mathrm{~N}^{+} \mathrm{Br}_{3}{ }^{-}$under mild and homogenous conditions at room temperature.

Therefore, in this article, we report the efficient trimethylsilylation of different types of hydroxyl groups including primary, secondary, hindered secondary, and substituted phenols using HMDS (I) in the presence of a catalytic amount of $\mathrm{PhMe}_{3} \mathrm{~N}^{+} \mathrm{Br}_{3}^{-}$(II) in dichloromethane at room temperature with good to excellent yields (Scheme 1 and Table 1).

As is evident from Table 1 a good range of turnover number (TON) and turnover frequency (TOF) for the catalyst is observed. To investigate the role of $\mathrm{PhMe}_{3} \mathrm{~N}^{+} \mathrm{Br}_{3}{ }^{-}$as the catalyst, benzhydrol was subjected to the trimethylsilylation reaction in the absence of a catalyst. However, no product was observed after $22 \mathrm{~h}$ (Table 1, entry 9).

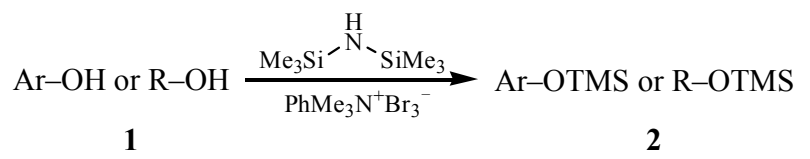<smiles>O=[N+]([O-])c1ccc(CO)cc1</smiles>

1b<smiles>CCCCCCCO</smiles>

19<smiles>OC(c1ccccc1)c1ccccc1</smiles>

$1 \mathrm{~h}$<smiles>OC1C2CC3CC(C2)CC1C3</smiles>

11

$1 \mathrm{~m}$<smiles>OCc1ccc(Cl)cc1Cl</smiles>

1c

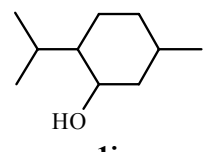

$1 \mathrm{i}$

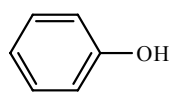

1n<smiles>CC(C)c1ccc(O)cc1</smiles>

$1 q$

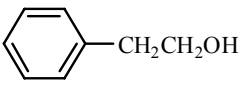

1d<smiles>O=C(c1ccccc1)C(O)c1ccccc1</smiles>

$\mathbf{1 j}$<smiles>CCCCCO</smiles>

$1 \mathrm{e}$

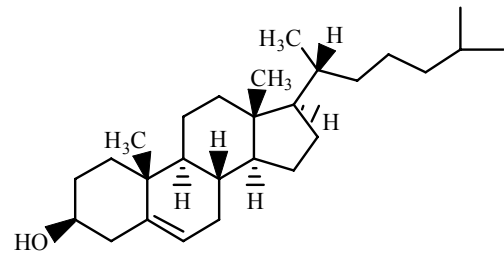

$1 \mathbf{k}$
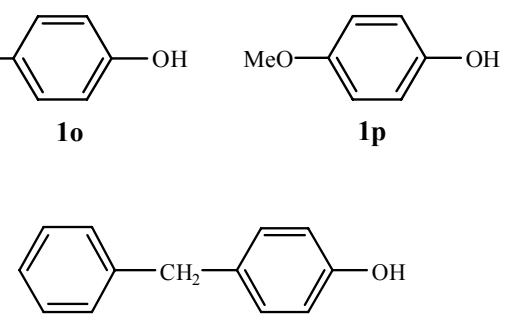

1s

1r

Scheme 1. Trimethylsilylation of different types of hydroxyl groups. 
Table 1 Trimethylsilylation of alcohols and phenols (1) to the corresponding trimethylsilyl alcohols and phenols (2) using HMDS (I) in the presence of a catalytic amount of $\mathrm{PhMe}_{3} \mathrm{~N}^{+} \mathrm{Br}_{3}^{-}$(II) in $\mathrm{CH}_{2} \mathrm{Cl}_{2}$ at room temperature

\begin{tabular}{|c|c|c|c|c|c|c|c|c|}
\hline \multirow{2}{*}{ Entry } & \multirow{2}{*}{ Substrate } & \multirow{2}{*}{ Product } & \multicolumn{2}{|c|}{ Substrate/HMDS/Cat. (mmol) } & \multirow{2}{*}{ Time (min) } & \multirow{2}{*}{ Yield $^{\mathrm{a}}(\%)$} & \multirow{2}{*}{ TON } & \multirow{2}{*}{ TOF $\left(\min ^{-1}\right)$} \\
\hline & & & I & II & & & & \\
\hline 1 & 1a & $2 a$ & 2 & 0.05 & 30 & 91 & 18.20 & 0.61 \\
\hline 2 & $1 b$ & $2 \mathbf{b}$ & 2 & 0.07 & 360 & 90 & 12.86 & 0.04 \\
\hline 3 & $1 \mathrm{c}$ & $2 c$ & 2 & 0.05 & 180 & 89 & 17.80 & 0.99 \\
\hline 4 & 1d & 2d & 2 & 0.05 & 5 & 94 & 18.80 & 3.76 \\
\hline 5 & $1 \mathrm{e}$ & $2 \mathrm{e}$ & 2 & 0.05 & 10 & 99 & 19.80 & 1.98 \\
\hline 6 & 1f & $2 f$ & 2 & 0.05 & 66 & $100^{\mathrm{b}}$ & 20.00 & 0.30 \\
\hline 7 & $1 \mathrm{~g}$ & $2 \mathrm{~g}$ & 2 & 0.05 & 105 & 86 & 17.20 & 0.16 \\
\hline 8 & $1 \mathrm{~h}$ & $2 \mathrm{~h}$ & 2 & 0.05 & 20 & 93 & 18.60 & 0.93 \\
\hline 9 & $1 \mathrm{~h}$ & $2 \mathrm{~h}$ & 2 & $-^{c}$ & 1320 & $-^{\mathrm{d}}$ & - & - \\
\hline 10 & $1 \mathrm{i}$ & $2 \mathbf{i}$ & 2 & 0.05 & 60 & 78 & 15.60 & 0.26 \\
\hline 11 & $1 \mathrm{j}$ & $2 \mathrm{j}$ & 2 & 0.05 & 91 & 97 & 19.40 & 0.21 \\
\hline 12 & $1 \mathrm{k}$ & $2 k$ & 2 & 0.07 & 140 & 90 & 12.86 & 0.09 \\
\hline 13 & 11 & 21 & 2 & 0.05 & 360 & 93 & 18.60 & 0.05 \\
\hline 14 & $1 \mathrm{~m}$ & $2 m$ & 2 & 0.05 & 1200 & - & - & - \\
\hline 15 & $1 n$ & $2 n$ & 2 & 0.05 & 17 & 88 & 17.60 & 1.04 \\
\hline 16 & 10 & 20 & 2 & 0.05 & 35 & 91 & 18.20 & 0.52 \\
\hline 17 & $1 p$ & $2 p$ & 2 & 0.05 & 12 & 90 & 18.00 & 1.50 \\
\hline 18 & $1 q$ & $2 q$ & 2 & 0.05 & 10 & 86 & 17.20 & 1.72 \\
\hline 19 & $1 \mathrm{r}$ & $2 r$ & 2 & 0.05 & 26 & 95 & 19.00 & 0.73 \\
\hline 20 & $1 \mathrm{~s}$ & $2 s$ & 2 & 0.05 & 26 & 98 & 19.60 & 0.75 \\
\hline
\end{tabular}

Reaction conditions: substrate $1 \mathrm{mmol}, \mathrm{CH}_{2} \mathrm{Cl}_{2} 10 \mathrm{ml}$, room temperature. ${ }^{\mathrm{a}}$ Isolated yield. ${ }^{\mathrm{b}}$ Conversion. ${ }^{\mathrm{c}} \mathrm{In}$ the absence of catalyst. ${ }^{\mathrm{d}}$ No reaction.
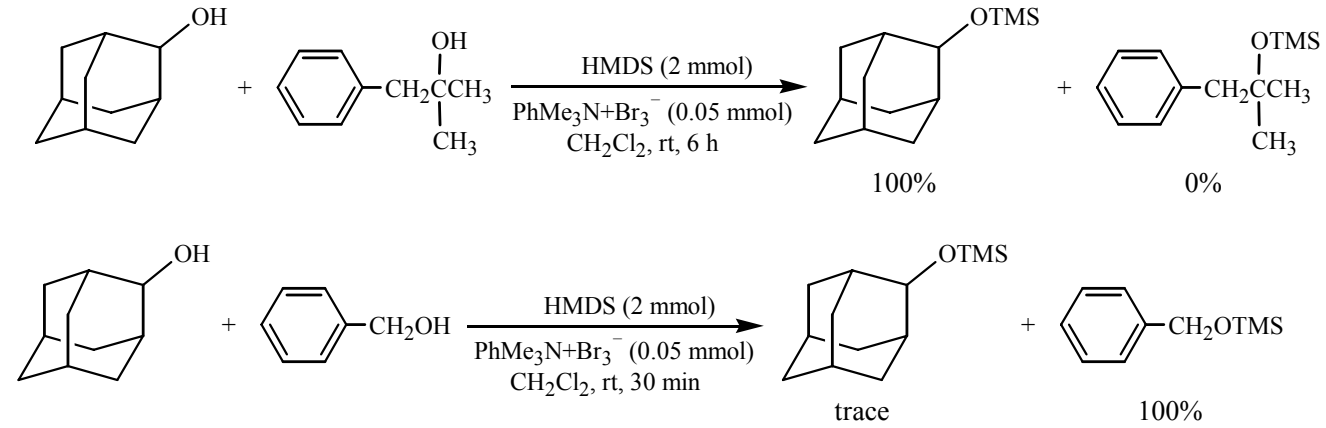

Scheme 2. Comparasion of reactivity of unhindered alcohols and hindered alcohols.

Also, our results show that unhindered alcohols are more reactive than hindered alcohols and the selectivity is out-

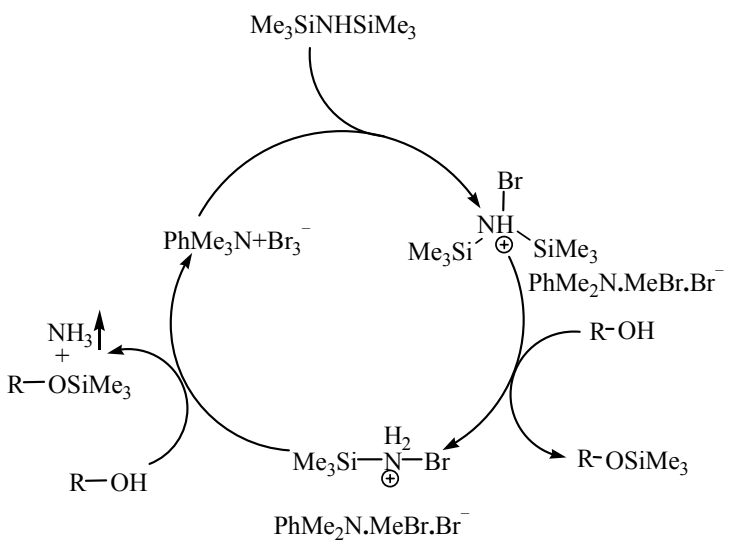

Scheme 3. Possible mechanism for trimethylsilylation of alcohols. lined in Scheme 2.

A plausible mechanism for trimethylsilylation is shown in Scheme 3. Phenyltrimethylammonium tribromide brominates hexamethyldisilazane, which polarizes the $\mathrm{Si}-\mathrm{N}$ bond. The polarization of the $\mathrm{Si}-\mathrm{N}$ bond converts HMDS to an activated silylating agent. Finally, the hydroxyl group silylates and ammonia is released as a by-product.

\section{Conclusions}

We report on a new catalytic method for the efficient trimethylsilylation of alcohol and phenol derivatives under metal-free, mild, and homogeneous conditions. This method offers shorter reaction time, high selectivity, non-toxic conditions, cost effective reagents and catalyst, and an easy workup. 


\section{References}

1 Kolvari E, Ghorbani-Choghamarani A, Salehi P, Shirini F, Zolfigol M A. J Iran Chem Soc, 2007, 4: 126

2 Greene T W, Wuts P G M. Protective Groups in Organic Synthesis. 3rd Ed. New York: John Wiley \& Sons, 1999

3 Isobe T, Fukuda K, Araki Y, Ishikaw T. Chem Commun, 2001: 243

4 Suginome M, Matsumoto A, Ito Y. J Org Chem, 1996, 61: 4884

5 Ito H, Takagi K, Miyahara T, Sawamura M. Org Lett, 2005, 7: 3001

6 Curhi M, Epifano F, Marcotullio M C, Rosati O, Costantino U. Synthetic Commun, 1999, 29: 541

7 Shirakawa E, Hironaka K, Otsuka H, Hayashi T. Chem Commun, 2006: 3927

8 Khazaei A, Rostami A, Rahmati S, Mahboubifar M. Phosphorus Sulfur Silicon, 2007, 182: 537

9 Shirini F, Zolfigol M A, Paktinat M. Synthesis, 2006: 4252

10 Shirini F, Mollarazi E. Catal Commun, 2007, 8: 1393

11 Ghorbani-Choghamarani A, Zolfigol M A, Hajjami M, Jafari S. J Chin Chem Soc, 2008, 55: 1208

12 Ghorbani-Choghamarani A, Amani K, Zolfigol M A, Hajjami M, Ayazi-Nasrabadi R. J Chin Chem Soc, 2009, 56: 255

13 Shirini F, Zolfigol M A, Abri A R. Monatsh Chem, 2008, 139: 17
14 Bideau F L, Henique J, Samuel E, Coradin T. Chem Commun, 2001: 1408

15 Tanabe Y, Okumura H, Madeda A, Murakami M. Tetrahedron Lett, 1994, 35: 8413

16 Biffis A, Castello E, Zecca M, Basato M. Tetrahedron, 2001, 57: 10391

17 Shibata T, Kanda K, Ueno Y, Fujiwara R. Bull Chem Soc Jpn, 2006, 79: 1146

18 Amantini D, Fringuelli F, Pizzo F, Vaccaro L. J Org Chem, 2001, 66: 6734

19 Ghorbani-Choghamarani A, Goudarziafshar H, Nikoorazm M, Yousefi S. Can J Chem, 2009, 87: 1144

20 Ghorbani-Choghamarani A, Goudarziafshar H, Nikoorazm M, Yousefi S. Lett Org Chem, 2009, 6: 335

21 Ghorbani-Choghamarani A, Nikoorazm M, Goudarziafshar H, Shiri L, Chenani Z. Bull Korean Chem Soc, 2009, 30: 972

22 Ghorbani-Choghamarani A, Shiri L, Zeinivand J. Bull Korean Chem Soc, 2008, 29: 2496

23 Ghorbani-Choghamarani A, Nikoorazm M, Goudarziafshar H, Tahmasbi B. Bull Korean Chem Soc, 2009, 30: 1388

24 Zolfigol M A, Amani K, Ghorbani-Choghamarani A, Hajjami M, Ayazi-Nasrabadi R, Jafari S. Catal Commun, 2008, 9: 1739

25 Habibi D, Zolfigol M A, Safaiee M, Shamsian A, GhorbaniChoghamarani A. Catal Commun, 2009, 10: 1257

26 Ghorbani-Choghamarani A, Zolfigol M A, Hajjami M, Rastgoo S, Mallakpour S. Lett Org Chem, 2010, 7: 249 